\author{
Peter H. Egbers \\ Renske Bultsma \\ Harmen Middelkamp \\ E. Christiaan Boerma
}

\section{Enabling speech in ICU patients during mechanical ventilation}

Accepted: 18 April 2014

Published online: 7 May 2014

(c) The Author(s) 2014. This article is published with open access at

Springerlink.com

\section{Dear Editor,}

Inability to speak during mechanical ventilation (MV) increases psychoemotional distress during ICU treatment [1]. Although relevant for all ICU patients, this problem becomes more prominent in patients with a prolonged weaning period. One way to enable speech in tracheotomised patients during the weaning period is to alternate MV with time-limited trials of cuff-deflated spontaneous breathing in combination a speaking valve. However, not all patients tolerate such interruptions of MV [2]. Although not commonly used, MV with deflated cuff in tracheotomised patients may be an alternative approach, and has been provided safely and comfortably in selected groups of non-ICU patients [3]. Its use has been associated with

improved swallowing and the possibility to speak [4]. However, in daily clinical ICU practise the use of MV under such conditions is limited by multiple ventilator alarms, as a result of air leakage. Furthermore, optimisation of speech is hampered by loss of airflow during open airway MV. We describe our experiences to improve speech in tracheotomised ICU patients during cuff-deflated $\mathrm{MV}$, in combination with a high flow non-invasive ventilator and a oneway tracheostomy speaking valve.

In 2013, 24 patients in our mixed medical-surgical ICU were tracheotomised, using a percutaneous dilatation technique. Five patients were able to wean and speak with progressive periods of spontaneous breathing trials. Five patients were weaned from MV without the ability to speak, one patient died within 2 days after the tracheotomy, and one patient had persistent insufficient air leakage around the cannula, despite reduction in cannula size. Table 1 summarizes the characteristics of the remaining 12 patients. Within $24 \mathrm{~h}$ after the tracheotomy procedure air leakage was present after deflation of the cuff. Invasive pressure support ventilation was replaced by a BiPAP mode with a non-invasive ventilator (BiPAP Vision ${ }^{\circledR}$, Philips, Best, the Netherlands) at equal ventilator settings. A minimum PEEP level of $8 \mathrm{cmH}_{2} \mathrm{O}$ was maintained in order to prevent rebreathing of $\mathrm{CO}_{2}$. Furthermore, an increasing level of PEEP has been associated with a prolonged duration and improved quality of speech [5]. In case all expiratory flow passed through the oropharyngeal route, a Passy Muir ${ }^{\circledR}$ speaking valve (Passy Muir Inc., Irvine, USA) was installed in the circuit to enhance speech (Fig. 1). All 12 patients were able to speak in the first day after switching to the BiPAP setting, enabling effective communication with relatives and medical personnel. Quality and duration of speech improved over time. Effective weaning from MV was achieved in all patients by progressive intervals of CPAP. During CPAP the ability to speak was similar to that in the BiPAP mode. Median time from the beginning of speech to the end of MV was $12 \pm 6$ days.

We conclude that in a selected group of difficult-to-wean tracheotomised ICU patients the ability to

Table 1 Patient characteristics

\begin{tabular}{lllllr}
\hline Gender & Age (years) & APACHE II & Reason for admittance & $\begin{array}{c}\text { Ability to speak } \\
\text { (days of MV) }\end{array}$ \\
\hline 1 & Male & 39 & 27 & Abdominal sepsis & 6 \\
2 & Male & 70 & 29 & Abdominal sepsis & 17 \\
3 & Male & 84 & NA & MODS after cardiac surgery & 17 \\
4 & Female & 56 & 38 & Abdominal sepsis & 17 \\
5 & Female & 66 & 46 & Pneumosepsis & 18 \\
6 & Male & 77 & 23 & Respiratory failure & 10 \\
7 & Male & 78 & 30 & Pancreatitis & 9 \\
8 & Female & 54 & 27 & Sepsis (necrotising fasciitis) & 5 \\
9 & Male & 63 & 21 & Gastrointestinal bleeding & 3 \\
10 & Male & 71 & 14 & Respiratory failure & 6 \\
11 & Male & 72 & 22 & Abdominal sepsis & 10 \\
12 & Male & 64 & Respiratory failure &
\end{tabular}

$A P A C H E$ acute physiology and chronic health evaluation, $M V$ mechanical ventilation, $M O D S$ multiple organ dysfunction syndrome, $N A$ not applicable 


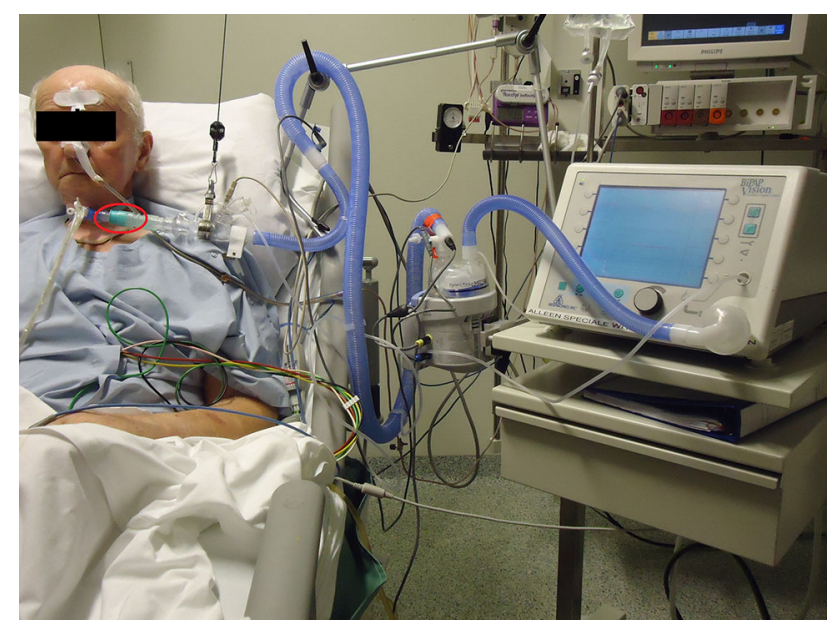

Fig. 1 Ventilator setting; Passy Muir ${ }^{\circledR}$ one-way speaking valve in red circle

speak may effectively be restored by cuff-deflated non-invasive BiPAP ventilation in combination with a one-way speaking valve. We encourage intensive care personnel to develop similar weaning protocols, in an effort to improve well-being of ICU patients.

Conflicts of interest On behalf of all the authors, the corresponding author states that there is no conflict of interest.

Open Access This article is distributed under the terms of the Creative Commons Attribution Noncommercial License which
2. Vitacca M, Callegari G, Sarva M, Bianchi L, Barbano L, Balbi B, Ambrosino N (2005) Physiological effects of meals in difficult-to-wean tracheostomised patients with chronic obstructive pulmonary disease. Intensive Care Med 31:236-242. doi: 10.1007/s00134-004-2530-Z

3. Bach JR, Alba AS (1990) Tracheostomy ventilation. A study of efficacy with deflated cuffs and cuffless tubes. Chest 97:679-683

4. Conway DH, Mackie C (2004) The effects of tracheostomy cuff deflation during continuous positive airway pressure. Anaesthesia 59:652-657. doi: 10.1111/j.1365-2044.2004.03755.x

5. Hoit JD, Banzett RB, Lohmeier HL, Hixon TJ, Brown R (2003) Clinical ventilator adjustments that improve speech. Chest 124:1512-1521

permits any noncommercial use, distribution, and reproduction in any medium, provided the original author(s) and the source are credited.

P. H. Egbers · R. Bultsma .

H. Middelkamp · E. C. Boerma (凶)

Medical Center Leeuwarden, Leeuwarden, Netherlands

e-mail: e.boerma@chello.nl

\section{References}

1. Khalaila R, Zbidat W, Anwar K, Bayya A, Linton DM, Sviri S (2011)

Communication difficulties and psychoemotional distress in patients receiving mechanical ventilation. Am J Crit Care 20:470-479. doi:10.4037/ajec2011989 